\title{
Milder Disease Stage in Patients With Primary Biliary Cholangitis Over a 44-Year Period: A Changing Natural History
}

Carla F. Murillo Perez, ${ }^{1,2}$ Jorn C. Goet (D), ${ }^{3}$ Willem J. Lammers, ${ }^{3}$ Aliya Gulamhusein, ${ }^{1}$ Henk R. van Buuren, ${ }^{3}$ Cyriel Y. Ponsioen, ${ }^{4}$ Marco Carbone, ${ }^{5}$ Andrew Mason, ${ }^{6}$ Christophe Corpechot, ${ }^{7}$ Pietro Invernizzi, ${ }^{5}$ Marlyn J. Mayo, ${ }^{8}$ Pier Maria Battezzati, ${ }^{9}$ Annarosa Floreani, ${ }^{10}$ Albert Pares, ${ }^{11}$ Frederik Nevens, ${ }^{12}$ Kris V. Kowdley, ${ }^{13}$ Tony Bruns (D), ${ }^{14}$ George N. Dalekos, ${ }^{15}$ Douglas Thorburn, ${ }^{16}$ Gideon Hirschfield, ${ }^{17}$ Nicholas F. LaRusso, ${ }^{18}$ Keith D. Lindor, ${ }^{19}$ Kalliopi Zachou, ${ }^{15}$ Raoul Poupon, ${ }^{7}$ Palak J. Trivedi, ${ }^{17}$ Xavier Verhelst, ${ }^{20}$ Harry L.A. Janssen, ${ }^{1}$ and Bettina E. Hansen ${ }^{1,3,21}$; on behalf of the GLOBAL PBC Study Group

\begin{abstract}
Changes over time in the presenting features and clinical course of patients with primary biliary cholangitis are poorly described. We sought to describe temporal trends in patient and disease characteristics over a 44-year period across a large international primary biliary cholangitis cohort of 4,805 patients diagnosed between 1970 and 2014, from 17 centers across Europe and North America. Patients were divided into five cohorts according to their year of diagnosis: 1970-1979 (n = 143), 1980-1989 ( $\mathrm{n}=858), 1990-1999$ ( $\mathrm{n}=1,754), 2000-2009(\mathrm{n}=1,815)$, and $\geq 2010(\mathrm{n}=235)$. Age at diagnosis, disease stage, response to ursodeoxycholic acid, and clinical outcomes were compared. Mean age at diagnosis increased incrementally by 2-3 years per decade from $46.9 \pm 10.1$ years in the 1970 s to $57.0 \pm 12.1$ years from 2010 onward $(P<0.001)$. The female to male ratio $(9: 1)$ and antimitochondrial antibody positivity $(90 \%)$ were not significantly variable. The proportion of patients presenting with mild biochemical disease (according to Rotterdam staging) increased from $41.3 \%$ in the 1970 s to $72.2 \%$ in the $1990 \mathrm{~s}(P<0.001)$ and remained relatively stable thereafter. Patients with a mild histological stage at diagnosis increased from $60.4 \%(1970-1989)$ to $76.5 \%(1990-2014)(P<0.001)$. Correspondingly, response to ursodeoxycholic acid according to Paris-I criteria increased; $51.7 \%$ in the 1970 s and $70.5 \%$ in the 1990 s $(P<0.001)$. Recent decades were also characterized by lower decompensation rates $(18.5 \%$ in the 1970 s to $5.8 \%$ in the 2000 s, $P<0.001)$ and higher 10 -year transplant-free survival $(48.4 \%, 68.7 \%, 79.7 \%$, and $80.1 \%$ for each respective cohort; $P<0.001)$. Conclusion: In recent decades, a pattern of primary biliary cholangitis presentation consistent with an older age at diagnosis alongside reduced disease severity has been noted; the observed trends may be explained by an increase in routine testing of liver function and/or a changing environmental trigger. (HEPATOLOGY 2018;67:1920-1930).
\end{abstract}

$\mathrm{P}$ rimary biliary cholangitis $(\mathrm{PBC})$ is a chronic autoimmune liver disease characterized by inflammation and destruction of the small intralobular bile ducts. ${ }^{(1-3)}$ The disease mainly affects middle-aged women and has a slow, progressive course that may lead to fibrosis, cirrhosis, and liver failure requiring liver transplantation. The standard

treatment for PBC is ursodeoxycholic acid (UDCA) as its long-term use improves liver biochemistry, delays histological progression, and may improve transplant-free survival. ${ }^{(4-6)}$ However, up to $40 \%$ of patients can have an inadequate response to UDCA that is associated with reduced transplant-free survival. $^{(4,7-9)}$

Abbreviations: $A M A$, antimitochondrial antibody; CI, confidence interval; HCC, hepatocellular carcinoma; HR, hazards ratio; IQR, interquartile range; $P B C$, primary biliary cholangitis; $S D$, standard deviation; UDCA, ursodeoxycholic acid.

Received July 17, 2017; accepted December 2, 2017.

Additional Supporting Information may be found at onlinelibrary. wiley.com/doi/10.1002/hep.29717/suppinfo.

Supported by unrestricted grants from Intercept Pharmaceuticals, Zambon Nederland B.V., and by the Foundation for Liver and Gastrointestinal Research (a not-for-profit foundation) in Rotterdam. The Netherlands. The supporting parties had no influence on the study design, data collection and analyses, writing of the manuscript, or the decision to submit the manuscript for publication. 
$\mathrm{PBC}$ is a rare disease, with multiple studies reporting an increase in its incidence and prevalence in recent years. ${ }^{(10-18)}$ In a systematic review conducted by Boonstra et al., ${ }^{(12)}$ the incidence of PBC varied from 0.33 to 5.8 per $100,000 /$ year, yet its temporal trends are conflicting as some studies suggest an increase, ${ }^{(11,12)}$ while others do not substantiate this finding. ${ }^{(19,20)}$ The prevalence ranged from 1.91 to 40.2 per 100,000 , and all investigated studies reported an increase. ${ }^{(10)}$ An increase in prevalence impacts how PBC contributes to the health care system and may be a result of multiple societal and disease factors. It is important to note that initial reports of an increasing prevalence began during the off-label use of UDCA period, which suggests that the increased prevalence in the UDCA era may be due to prolonged survival. ${ }^{(11,14,16)}$ Correspondingly, the absolute number of liver transplantations for PBC has decreased in Europe and the United States since the introduction of UDCA in the early 1990s. ${ }^{(3,9,21-23)}$
In addition to epidemiological changes, the clinical presentation of $\mathrm{PBC}$ has changed over the years. Whereas most patients presented with an advanced histological stage in earlier decades, nowadays most patients present during an asymptomatic stage. ${ }^{(24,25)}$ Therefore, the underlying assumption that PBC, as a disease, is a static entity may not be accurate. We used a representative large cohort of patients with $\mathrm{PBC}$ to assess how disease presentation and prognosis have changed over the last nearly 50 years. In doing so, we provide long-term insight into the changing nature of $\mathrm{PBC}$ in clinical practice.

\section{Patients and Methods POPULATION AND STUDY DESIGN}

This was a retrospective study based on patient data retrieved from the Global PBC Study Group database, the characteristics of which have been described in

Copyright $(02017$ by the American Association for the Study of Liver Diseases.

View this article online at wileyonlinelibrary.com.

DOI 10.1002/hep.29717

Potential conflict of interest: Dr. Pares consults for Intercept and Novartis. Dr. Mason consults for, is on the speakers' bureau of, and received grants from Intercept. He received grants from GlaxoSmithKline and Novartis. Dr. Corpechot consults for Intercept. Dr. Ponsioen consults for, is on the speakers' bureau of, and received grants from Takeda. He consults for and is on the speakers' bureau of AbbVie. He is on the speakers' bureau of Dr. Falk. Prof. Janssen consults for and received grants from AbbVie, Bristol-Myers Squibb, Gilead, Janssen, MedImmune, Merck, and Roche. He consults for Benitec and Arbutus. He received grants from Innogenetics and Medtronic. Dr. Mayo received grants from Intercept. Dr. Bruns consults for, advises, and received grants from Intercept. He received grants and honoraria from Falk. He received grants from Gilead. Dr. Lammers consults for Intercept. Dr. Kowdley consults for, advises, is on the speakers' bureau of, and received grants from Gilead and Intercept. He advises and received grants from Novartis. He received grants from Genfit and GlaxoSmithKline.

\section{ARTICLE INFORMATION:}

From the ${ }^{1}$ Toronto Centre for Liver Disease, Toronto General Hospital, University Health Network, Toronto, ON, Canada; ${ }^{2}$ Insitute of Medical Science, University of Toronto, Toronto, ON, Canada; ${ }^{3}$ Gastroenterology and Hepatology, Erasmus University Medical Center, Rotterdam, The Netherlands; ${ }^{4}$ Department of Gastroenterology and Hepatology, Academic Medical Center, Amsterdam, The Netherlands; ${ }^{5}$ Division of Gastroenterology and Center for Autoimmune Liver Diseases, Department of Medicine and Surgery, University of MilanBicocca, Milan, Italy; ${ }^{6}$ Divison of Gastroenterology and Hepatology, University of Alberta, Edmonton, AB, Canada; ${ }^{7}$ Centre de Référence des Maladies Inflammatoires des VoiesBiliaires, Hôpital Saint-Antoine, Paris, France; ${ }^{8}$ Digestive and Liver diseases, UT Southwestern Medical Center, Dallas, TX; ${ }^{9}$ Department of Health Sciences, Università degli Studi di Milano, Milan, Italy; ${ }^{10}$ Department of Surgery, Oncology and Gastroenterology, University of Padua, Padua, Italy; ${ }^{11}$ Liver Unit, Hospital Clínic, CIBERehd, IDIBAPS, University of Barcelona, Barcelona, Spain; ${ }^{12}$ Department of Hepatology, University Hospitals Leuven, KU Leuven, Leuven, Belgium; ${ }^{13}$ Liver Care Network, Swedish Medical Center, Seattle, WA; ${ }^{14}$ Department of Internal Medicine IV, Jena University Hospital, Friedrich Schiller University of Jena, Jena, Germany; ${ }^{15}$ Department of Medicine and Research Laboratory of Internal Medicine, University of Thessaly, Larissa, Greece; ${ }^{16}$ The Sheila Sherlock Liver Centre, The Royal Free Hospital, London, UK; ${ }^{17}$ NIHR, Biomedical Research Centre and Centre for Liver Research, University of Birmingham, Birmingham, UK; ${ }^{18}$ Division of Gastroenterology and Hepatology, Mayo Clinic, Rochester, MN; ${ }^{19}$ Arizona State University, Phoenix, AZ; ${ }^{20}$ Department of Gastroenterology and Hepatology, Ghent University Hospital, Ghent, Belgium;

${ }^{21}$ Institute of Health Policy, Management and Evaluation, University of Toronto, Toronto, ON, Canada.

\section{ADDRESS CORRESPONDENCE AND REPRINT REQUESTS TO:}

Bettina E. Hansen, M.Sc., Ph.D.

Toronto Centre for Liver Disease, Toronto General Hospital 200 Elizabeth Street
Toronto, ON, M5G 2C4, Canada

E-mail: Bettina.hansen@utoronto.ca

Tel: +1-416-340-5157 
previous publications. ${ }^{(26,27)}$ The database comprises long-term follow-up cohorts from 17 centers across North America and Europe. UDCA-treated and nontreated patients aged $\geq 18$ with an established $\mathrm{PBC}$ diagnosis from 1970 to 2014, according to internationally accepted guidelines, were included in the study. ${ }^{(3,28,29)}$ Patients with either a short follow-up ( $<6$ months), an unknown date of important clinical events, an overlap syndrome, or another concomitant liver disease were excluded. Completeness and accuracy of the database were established through visits to individual centers. This study was conducted in accordance with the 1975 Declaration of Helsinki. The protocol was approved by the institutional research board of the corresponding center and at all participating centers per local regulations.

\section{DATA COLLECTION}

In the established database, study entry (baseline) was the date of UDCA therapy initiation or the date of first visit for nontreated patients. The following demographic and clinical data were available at study entry: sex, date of birth, date of diagnosis, antimitochondrial antibody (AMA) serological status, liver histology, biochemical disease stage, and UDCA therapy (if received and dosage). In addition, the following laboratory values were available at study entry and every 6-12 months until the end of follow-up: alkaline phosphatase, aspartate aminotransferase, alanine aminotransferase, total bilirubin, albumin, and platelet count. Histology was considered if the liver biopsy was completed within 24 months of diagnosis date and dichotomized according to Ludwig et al.'s ${ }^{(30)}$ and Scheuer's ${ }^{(31)}$ classification, specifically as mild (stages I and II) and advanced (stages III and IV). The Rotterdam criteria were used to determine patients' biochemical stage. According to these criteria, mild stage is defined as normal bilirubin and albumin, moderate stage is defined as abnormal bilirubin or albumin, and advanced stage is defined as abnormal bilirubin and albumin. ${ }^{(9,32)}$ Baseline aspartate aminotransferase/ platelet ratio index, an independent predictor of transplant-free survival, was calculated to stratify patients at risk of liver transplantation and death based on a threshold of $0.54 .^{(33)}$ The first occurrence of hepatic decompensation (ascites, variceal bleeding, or hepatic encephalopathy), hepatocellular carcinoma (HCC), liver transplantation, or all-cause mortality was also retrieved.
In patients who received therapy, biochemical response to UDCA was determined according to Barcelona, Paris-I, Rotterdam, Toronto, and Paris-II criteria. $^{(7-9,34,35)}$ In addition, the GLOBE score was compared to age-specific thresholds to determine UDCA response. ${ }^{(26)}$ Patients were considered responders if their GLOBE score did not surpass their age-specific threshold.

\section{STATISTICAL ANALYSIS}

Patients diagnosed between 1970 and 2014 were divided into five cohorts according to their year of diagnosis: 1970-1979, 1980-1989, 1990-1999, 20002009 , and $\geq 2010$. To compare patient and disease characteristics across the five cohorts, we conducted chi-squared tests for categorical variables and analyses of variance for continuous data. $P<0.05$ was considered significant for all statistical analyses. Significant results were further analyzed to correct for any possible confounding variables and to assess the influence of other explanatory variables on the outcome measure. A multivariable logistic regression was applied to binary outcomes, such as biochemical response to UDCA, biochemical disease stage (moderate and advanced disease stage grouped as advanced), and histological stage (odds ratio with 95\% confidence interval [CI]).

For time-to-event analyses, patients diagnosed from 2010 onward were excluded due to a shorter follow-up period than the other cohorts. Patients without an event and those who were lost to follow-up were censored at their last visit. The rates of hepatic decompensation, HCC, and liver transplant-free survival were assessed by Kaplan-Meier estimates and compared across decades using the log-rank test. If decompensation occurred within the first year of study entry, the patient was excluded from the time-to-event analysis for decompensation. Transplant-free survival of the PBC population was also compared within each decade to an age- and gender-matched Dutch population. These outcomes were also estimated by Cox proportional hazards' modeling (hazards ratio [HR] with 95\% CI).

Demographic and clinical characteristics are presented as count (percentage) for categorical data and mean \pm standard deviation (SD) for continuous variables. Laboratory values are presented as median (interquartile range $[\mathrm{IQR}]$ ). Data that were not normally distributed were log-transformed for the analyses. All analyses were two-sided and were performed using 
TABLE 1. Demographic and Clinical Characteristics of PBC Patients at Study Entry Over Calendar Time

\begin{tabular}{|c|c|c|c|c|c|c|}
\hline $\begin{array}{l}\text { Baseline } \\
\text { Characteristics }\end{array}$ & $\begin{array}{l}1970-1979 \\
(n=143)\end{array}$ & $\begin{array}{l}1980-1989 \\
(n=858)\end{array}$ & $\begin{array}{c}1990-1999 \\
(n=1754)\end{array}$ & $\begin{array}{l}2000-2009 \\
(n=1815)\end{array}$ & $\begin{array}{c}\geq 2010 \\
(n=235)\end{array}$ & $P$ \\
\hline Age at diagnosis (years)* & $46.9(10.1)$ & $50.1(10.7)$ & $52.8(11.5)$ & $55.0(12.5)$ & $57.0(12.1)$ & $<0.001$ \\
\hline Female & $131(91.6)$ & $775(90.3)$ & $1,593(90.8)$ & $1,619(89.2)$ & $207(88.1)$ & 0.396 \\
\hline AMA-positive $^{\dagger}$ & $123 / 140(87.9)$ & $763 / 842(90.6)$ & $1,565 / 1,704(91.8)$ & $1,599 / 1,765(90.6)$ & $213 / 235(90.6)$ & 0.449 \\
\hline Laboratory values ${ }^{\ddagger}$ & & & & & & \\
\hline Serum ALP (XULN) & $2.99(1.85-4.77)$ & $3.20(1.95-5.23)$ & $2.03(1.30-3.56)$ & $1.79(1.19-3.05)$ & $1.55(1.08-2.93)$ & $<0.001$ \\
\hline Serum bilirubin $(\times U L N)$ & $0.93(0.60-2.1)$ & $0.81(0.52-1.30)$ & $0.64(0.47-1.00)$ & $0.60(0.41-0.95)$ & $0.59(0.41-1.0)$ & $<0.001$ \\
\hline Serum AST $(\times U L N)$ & $1.59(1.06-2.32)$ & $1.95(1.20-2.77)$ & $1.35(0.87-2.20)$ & $1.30(0.90-2.00)$ & $1.29(0.85-2.07)$ & $<0.001$ \\
\hline Serum ALT (XULN) & $1.30(0.85-2.47)$ & $2.00(1.3-3.1)$ & $1.66(1.03-2.68)$ & $1.42(0.90-2.27)$ & $1.32(0.75-2.38)$ & $<0.001$ \\
\hline Serum albumin $(\times \operatorname{LLN})$ & $1.11(0.99-1.21)$ & $1.16(1.06-1.26)$ & $1.14(1.06-1.23)$ & $1.14(1.06-1.23)$ & $1.14(1.03-1.23)$ & 0.005 \\
\hline Platelet count $\left(\times 10^{9} / \mathrm{L}\right)$ & $194(127-242.5)$ & $224(165-275)$ & $238(185-289)$ & $258(204-311)$ & $237(174.5-291)$ & $<0.001$ \\
\hline APRI $(>0.54)^{\S}$ & $61(76.3)$ & $260(69.0)$ & $456(52.3)$ & $476(47.4)$ & $85(54.1)$ & $<0.001$ \\
\hline Biochemical disease stage $\|$ & $121 / 143$ & $627 / 859$ & $985 / 1,755$ & $1,073 / 1,816$ & $152 / 235$ & $<0.001$ \\
\hline Mild & $50 / 121(41.3)$ & $370 / 627(59.0)$ & $711 / 985(72.2)$ & $757 / 1,073(70.5)$ & $106 / 152(69.7)$ & \\
\hline Moderately advanced & $51 / 121(42.1)$ & 196/627 (31.3) & $205 / 985(20.8)$ & $238 / 1,073(22.2)$ & $27 / 152(17.8)$ & \\
\hline Advanced & $20 / 121(16.5)$ & 61/627 (9.7) & 69/985 (7.0) & 78/1073 (7.3) & $19 / 152(12.5)$ & \\
\hline Histological disease stage $e^{q}$ & \multicolumn{2}{|c|}{$326 / 1001$} & $948 / 1754$ & \multicolumn{2}{|c|}{$943 / 2050$} & $<0.001$ \\
\hline Mild (I or II) & \multicolumn{2}{|c|}{$197(60.4)$} & $634(66.9)$ & \multicolumn{2}{|c|}{$721(76.5)$} & \\
\hline Advanced (III or IV) & \multicolumn{2}{|c|}{$129(39.6)$} & $314(33.1)$ & \multicolumn{2}{|c|}{$222(23.5)$} & \\
\hline UDCA-treated $\#$ & 78/139 (56.1) & $735 / 832(88.3)$ & $1,605 / 1,737(92.4)$ & $1,563 / 1,789(87.4)$ & $195 / 230(84.8)$ & $<0.001$ \\
\hline
\end{tabular}

Data presented as mean (SD), n (\%), or median (IQR).

*Age at diagnosis not available for one patient in 2000-2009 cohort.

${ }^{\dagger}$ AMA status was available for 4,686 (97.5\%) patients.

${ }^{\ddagger}$ ALP, bilirubin, AST, and ALT were log-transformed prior to analyses and availability for laboratory values is as follows: ALP, 3,560 (74.1\%); bilirubin, 3,595 (74.8\%); AST, 3,460 (72.0\%); ALT, 3,007 (62.6\%); albumin, 3,039 (63.2\%); platelet count, 2,769 (57.6\%).

${ }^{\S}$ The cutoff point for APRI $>0.54$ at baseline is predictive of liver transplantation or death. ${ }^{(33)}$

${ }$ Biochemical disease stage classification according to Rotterdam criteria ${ }^{(9)}$ was available in 2,958 (61.6\%) patients.

"Histological disease stage at diagnosis according to Ludwig et al. ${ }^{(30)}$ and Scheuer ${ }^{(31)}$ classification was available in 2,217 (46.1\%) patients.

\#UDCA therapy status was available for 4,727 patients (98.4\%).

Abbreviations: ALP, alkaline phosphatase; ALT, alanine aminotransferase; APRI, AST to platelet ratio index; AST, aspartate aminotransferase; LLN, lower limit of normal; ULN, upper limit of normal.

IBM SPSS Statistics for Windows, version 24.0 (IBM Corp., Armonk, NY).

\section{Results}

\section{STUDY POPULATION CHARACTERISTICS}

A total of 4,805 PBC patients, diagnosed between 1970 and 2014, were included and divided into five cohorts according to their year of diagnosis (Table 1; Supporting Table S1): 143 patients were diagnosed from 1970 to 1979,858 patients from 1980 to 1989 , 1,754 patients from 1990 to $1999,1,815$ patients from 2000 to 2009, and 235 patients from 2010 onward. The characteristics of each cohort are presented in Table 1. The median follow-up times for the five respective cohorts were 6.7 years (IQR 3.0-14.3), 8.9 years (IQR 4.0-14.7), 10.0 years (IQR 6.0-13.9), 5.6 years (IQR 3.4-8.3), and 1.6 years (IQR 1.0-2.1). The mean time from diagnosis to study entry was variable for each cohort: 11.1 years (SD 7.0) for the 1970s, 5.1 years (SD 4.5) for the 1980s, 1.4 years (SD 2.3) for the 1990s, 0.4 years (SD 1.1) for the 2000s, and 0.1 years (SD 0.2) from 2010 onward. To consider this variation, all analyses were repeated in a subgroup of patients $(\mathrm{n}=3,518)$ with a maximum 2-year lag between diagnosis and study entry, which included $14 \%, 29 \%, 76 \%, 93 \%$, and $100 \%$ of patients from the main analysis in each respective cohort (Supporting Table S2).

\section{AGE AND SEX TRENDS}

The mean age at diagnosis increased incrementally from $46.9 \pm 10.1$ years in the 1970 s to $57.0 \pm 12.1$ years from 2010 onward $(P<0.001)$ (Fig. 1A). This trend was consistent across center, sex, and biochemical disease stage (Supporting Fig. S1A-C). The effect of calendar time on the increase in age at diagnosis remained significant $(P<0.001)$ after correcting for sex (Supporting Table S3). Furthermore, the age distribution of patients notably changed over the investigated decades $(P<0.001)$ (Fig. 1B). The proportion of patients aged 50-59 years at diagnosis remained 

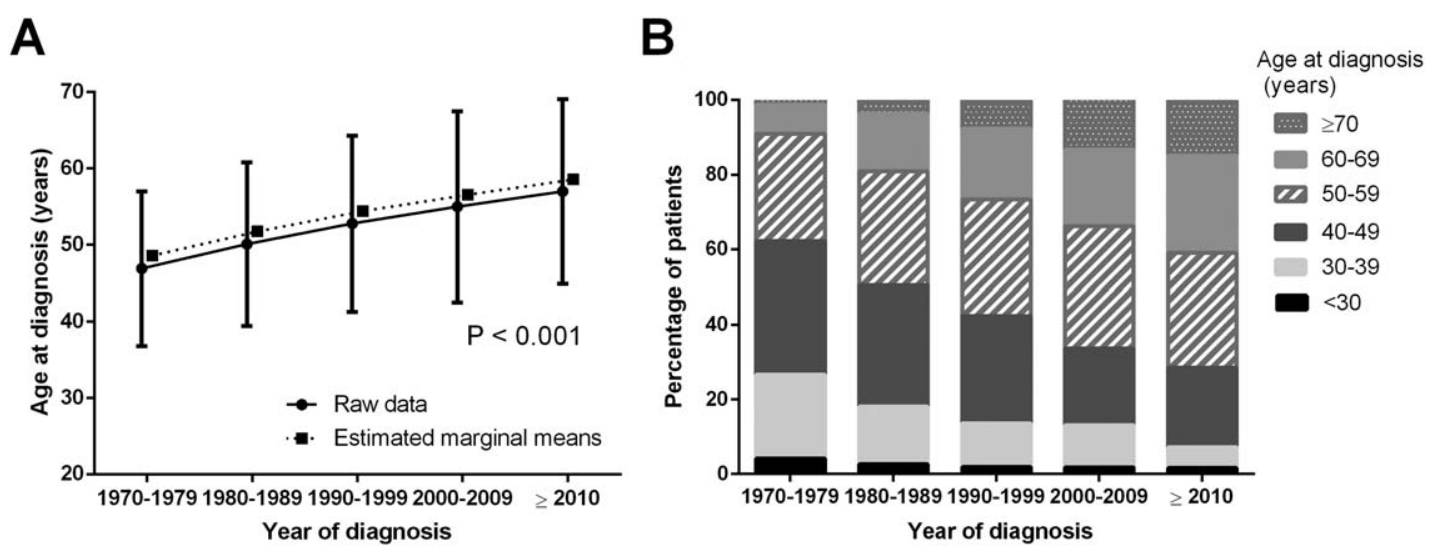

FIG. 1. Age at diagnosis of PBC patients across different decades. (A) Mean age ( \pm SD) at diagnosis (dots) and estimated marginal means (squares) obtained after adjusting for sex. (B) Distribution of age groups over calendar time.

relatively stable across the years, whereas the proportion of patients $<50$ years of age decreased and that of patients $\geq 60$ years of age increased. There was no significant temporal trend in the female to male ratio, which remained approximately 9:1 (Table 1).

\section{LIVER BIOCHEMISTRY AND SEROLOGICAL STATUS}

The proportion of patients who were AMA-positive did not significantly differ across the investigated decades (Table 1). Median alkaline phosphatase and bilirubin values (times the upper limit of normal) at study entry decreased, while circulating platelet counts were noted to increase $(P<0.001)$, which collectively suggests a less advanced disease stage. The proportion of patients with alkaline phosphatase values $<2$ times the upper limit of normal increased gradually from 30.0\% in the 1970s to $63.1 \%$ from 2010 onward $(P<0.001)$ (Fig. 2A). The proportion of patients with normal serum bilirubin concentrations also increased from $51.1 \%$ in the 1970 s to $77.6 \%$ in the 1990 s, after which it remained relatively stable $(P<0.001)$ (Fig. 2B). Furthermore, a reduced percentage of patients with aspartate aminotransferase/platelet ratio index $>0.54$ at study entry was observed (Table 1).

\section{TRENDS IN BIOCHEMICAL AND HISTOLOGICAL DISEASE STAGE}

There was a gradual increase in the proportion of patients presenting with a mild biochemical disease stage from the 1970s to 1990s and remained stable thereafter $(P<0.001)$ (Fig. 2C). In a multivariable logistic regression, calendar time was a significant predictor for biochemical disease stage $(P<0.001)$ after adjusting for sex and age at diagnosis. Earlier decades were associated with an advanced biochemical disease stage.

Out of 2,831 patients who underwent liver biopsy at diagnosis, 2,217 patients had histological disease stage available and were included in a subgroup analysis that combined cohorts due to the limited number of biopsies in the first and last cohorts. There were 326 biopsies from 1970 through 1989, 948 biopsies from 1990 through 1999, and 943 from 2000 through 2014. The proportion of patients with a mild histological stage (I or II) at diagnosis increased with time (Table 1 and Fig. 2D). In a multivariable logistic regression, calendar time was a significant predictor for histological stage after adjusting for sex and age at diagnosis $(P<$ 0.001).

\section{TRENDS IN UDCA RESPONSE RATES}

The proportion of patients who ever received UDCA increased across the investigated decades $(P<$ 0.001) (Table 1). In patients who received UDCA, the median number of years between diagnosis and the start of UDCA therapy decreased across the respective cohorts (1970s to $\geq 2010$ ): 12.6 years (IQR 10.616.1), 4.4 years (IQR 2.1-8.1), 0.23 years (IQR 0.02.0), 0.05 years (IQR $0.0-0.41$ ), and 0.0 years (IQR 0.0-0.04). Additionally, the median initial dosage of 
A

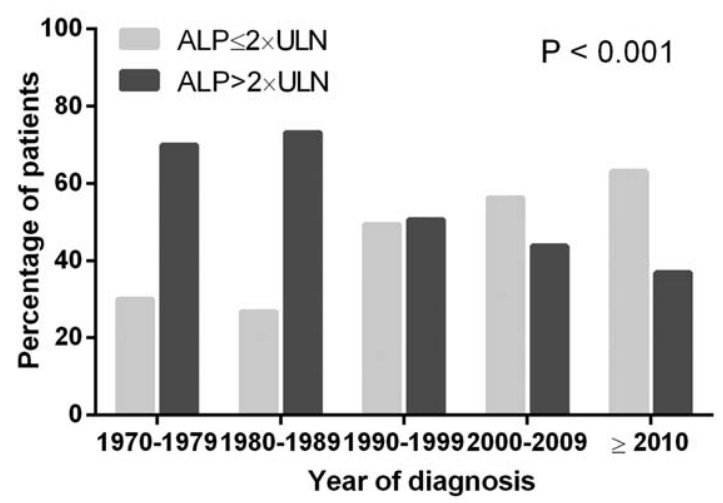

C

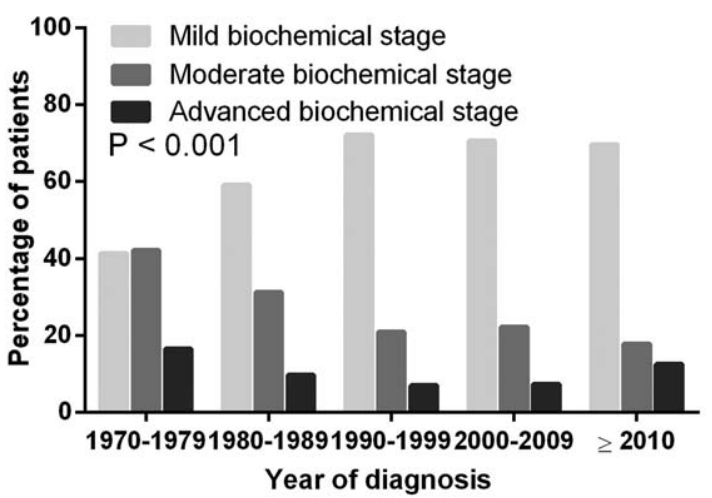

B

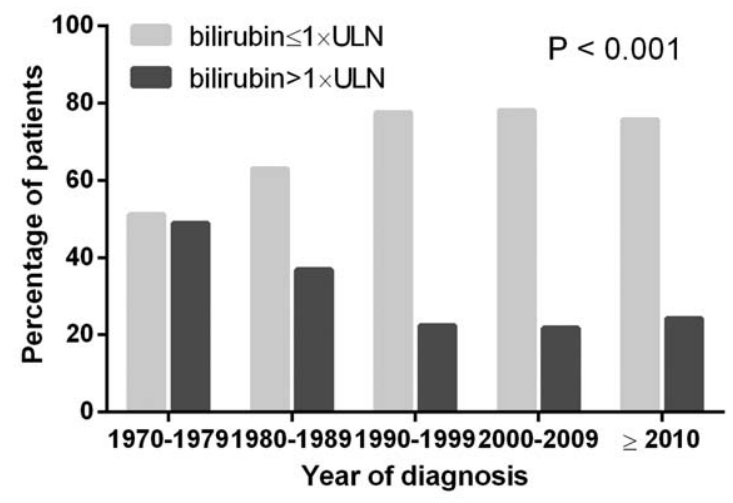

D

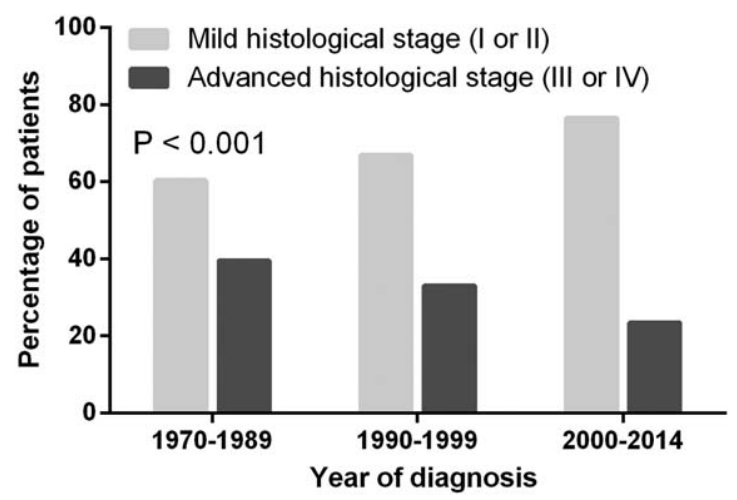

FIG. 2. Study entry characteristics associated with disease severity of patients diagnosed in different decades. (A) Percentage of patients with alkaline phosphatase above or below 2 times the upper limit of normal. (B) Percentage of patients with bilirubin above or below 1 times the upper limit of normal. (C) Percentage of patients corresponding to each biochemical stage according to Rotterdam criteria ${ }^{(9)}$ : mild (normal albumin and bilirubin), moderate (abnormal albumin or bilirubin), advanced (abnormal albumin and bilirubin). (D) Percentage of patients corresponding to each histological stage at diagnosis according to Ludwig et al.'s ${ }^{(30)}$ and Scheuer's ${ }^{(31)}$ classifications: mild (stages I and II) or advanced (stages III and IV). Abbreviations: ALP, alkaline phosphatase; ULN, upper limit of normal.

UDCA received by patients across the five respective cohorts increased: $9.4 \mathrm{mg} / \mathrm{kg} /$ day (IQR 8.5-11.0), 10.0 $\mathrm{mg} / \mathrm{kg} /$ day (IQR 8.7-13.7), $12.2 \mathrm{mg} / \mathrm{kg} /$ day (IQR 9.2-14.7), $13.5 \mathrm{mg} / \mathrm{kg} / \mathrm{day}$ (IQR 11.1-15.3), and 13.3 $\mathrm{mg} / \mathrm{kg} /$ day (IQR 11.1-15.1).

The proportion of UDCA responders according to Paris-I, Toronto, Paris-II, Rotterdam, and GLOBE score criteria increased over the investigated decades $(P<0.001)$ but not that according to Barcelona criteria (Fig. 3; Supporting Table S4). Importantly, this trend remained true in patients who did not meet the individual criteria at baseline (Supporting Table S5). In a multivariable logistic regression, calendar time was not a significant predictor for UDCA response according to Paris-I criteria (Table 2). Response was associated with an increased age at diagnosis and lower alkaline phosphatase and bilirubin levels $(P<0.001)$.
Additionally, calendar time was also not a significant predictor for UDCA response according to Toronto, Paris-II, Rotterdam, and GLOBE score criteria (results not shown).

\section{DECOMPENSATION, HCC, AND TRANSPLANT-FREE SURVIVAL}

The 10-year incidence rate of hepatic decompensation (ascites, variceal bleeding, or hepatic encephalopathy, whichever came first) decreased over time: $18.5 \%$ in the $1970 \mathrm{~s}, 13.7 \%$ in the $1980 \mathrm{~s}, 8.5 \%$ in the $1990 \mathrm{~s}$, and $5.8 \%$ in the 2000 s (Fig. 4Ai). All pairwise comparisons were significantly different, except the difference between the 1970s and 1980s cohorts $(P=0.45)$. In a multivariable Cox regression, a temporal trend of lower decompensation risk was observed after adjusting 


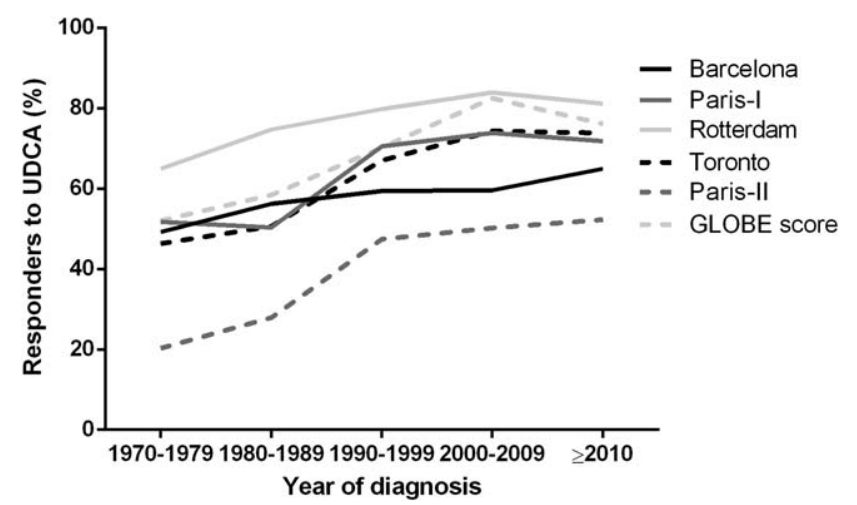

FIG. 3. Response rates to UDCA therapy over calendar time. Response was determined according to various published criteria: Barcelona, Paris-I, Rotterdam, Toronto, Paris-II, and the GLOBE score. ${ }^{(7-9,26,34,35)}$ Response rates according to all criteria were significantly different over calendar time $(P<0.001)$, except for Barcelona criteria $(P=0.19)$.

for sex and age at diagnosis (Fig. 4Bi) $(P=0.07)$. Calendar time as a continuous variable was a significant predictor for hepatic decompensation (HR, per 10-year increase, $0.57 ; 95 \%$ CI 0.44-0.75; $P<0.001$ ).

The 10-year HCC incidence rates across the investigated decades were $10.3 \%, 4.0 \%, 2.1 \%$, and $2.3 \%$,

TABLE 2. Multivariable Logistic Regression for the Attainment of Biochemical Response According to Paris-I* $(\mathrm{n}=2,283)$

\begin{tabular}{lccc} 
Variable & OR & $95 \% \mathrm{Cl}$ & $P$ \\
\hline $\begin{array}{l}\text { Male sex } \\
\text { Year of diagnosis }\end{array}$ & 0.90 & $0.63-1.29$ & 0.58 \\
$\quad$ 1970-1979 & 1.00 & & 0.67 \\
$\quad 1980-1989$ & 0.80 & $0.37-1.71$ & 0.66 \\
1990-1999 & 1.01 & $0.44-2.37$ & 0.96 \\
2000-2009 & 0.97 & $0.40-2.32$ & 0.94 \\
$\quad \geq 2010$ & 0.92 & $0.33-2.57$ & 0.88 \\
Age at diagnosis (years) & & & 0.04 \\
$\quad<30$ & 1.00 & & \\
30-39 & 1.29 & $0.53-3.15$ & 0.57 \\
40-49 & 1.41 & $0.60-3.33$ & 0.44 \\
50-59 & 1.95 & $0.82-4.59$ & 0.13 \\
60-69 & 2.06 & $0.86-4.96$ & 0.11 \\
$\quad \geq 70$ & 2.06 & $0.82-5.21$ & 0.13 \\
Log bilirubin $(\times U L N)$ & 0.01 & $0.01-0.02$ & $<0.001$ \\
Log ALP (XULN) & 0.12 & $0.08-0.18$ & $<0.001$ \\
Difference between diagnosis & 0.98 & $0.94-1.03$ & 0.44
\end{tabular}
and study entry (years)

*Response rate according to Paris-I is defined as ALP $\leq 3$ $\times \mathrm{ULN}, \mathrm{AST} \leq 2 \times \mathrm{ULN}$, and normal bilirubin after 1 year of UDCA therapy.

ALP, alkaline phosphatase; OR, odds ratio; ULN, upper limit of normal. respectively (Fig. 4Aii). The Kaplan-Meier estimate of cumulative HCC incidence was significantly higher in the 1970s compared to the 1980s $(P=0.01), 1990$ s $(P<0.001)$, and 2000s $(P<0.001)$. In a multivariable Cox regression, calendar time was not a significant predictor for HCC risk $(P=0.68)$ after adjusting for sex, age at diagnosis, and UDCA treatment (Fig. 4Bii).

The 10-year liver-related death rate decreased from 1970 through 2009: 34.6\%, 13.2\%, 5.6\%, and 6.4\% $(P<0.001)$. Furthermore, the 10 -year transplant-free survival rate improved over the four respective investigated decades: $48.4 \%, 68.7 \%, 79.7 \%$, and $80.1 \%$ (Fig. 4Aiii). There was a significant difference in transplantfree survival between the 1970s and 1980s $(P<0.001)$ and between the 1980s and 1990s $(P<0.001)$. However, the transplant-free survival rates between the 1990s and 2000s were equivalent $(P=0.80)$. In a multivariable Cox regression, calendar time remained an independent predictor of transplant-free survival, and earlier decades were associated with an increased risk for liver transplantation and all-cause mortality (Fig. 4Biii; Supporting Table S6). Furthermore, the 10-year transplant-free survival of $\mathrm{PBC}$ patients has improved even when compared to an age- and gender-matched general population (1970s: $\mathrm{HR}=4.38 ; 95 \%$ CI 3.54-5.43; $P<$ 0.001; 1980s: $\mathrm{HR}=2.90 ; 95 \%$ CI 2.60-3.24; $P<0.001 ;$ 1990s: HR $=2.14 ; 95 \%$ CI 1.94-2.36; $P<$ 0.001; 2000s: HR $=1.93 ; 95 \%$ CI 1.69-2.21; $P<$ $0.001)$.

\section{Discussion}

In this study of a large, internationally representative cohort of PBC patients, we demonstrate that patients diagnosed in recent decades are older and have a milder disease stage compared to patients diagnosed in earlier decades. In addition, more patients respond favorably to UDCA therapy and have improved transplant-free survival. These results provide unique insight into the possible changing natural history of $\mathrm{PBC}$ over the last five decades. It is noteworthy to mention that similar results have been observed in a study from Sweden that included 246 patients diagnosed with primary sclerosing cholangitis between 1984 and 2004. Bergquist et al. reported an increase in age at diagnosis and lower frequency of symptoms in patients diagnosed after 1998. ${ }^{(36)}$

Although some of the observed trends could be potentially attributed to more sensitive AMA tests that 
A

i

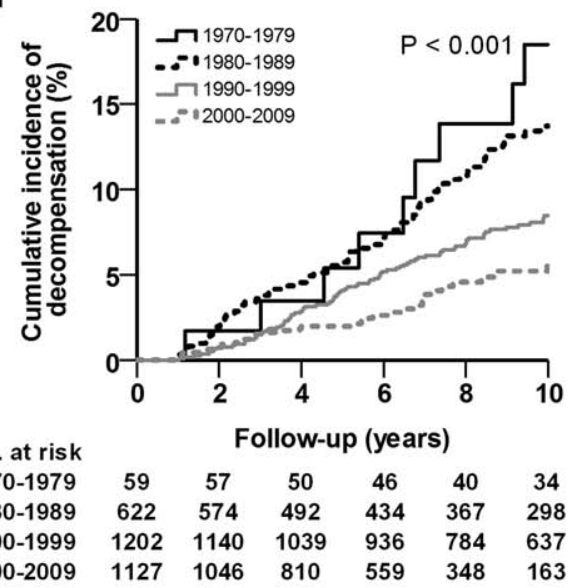

ii

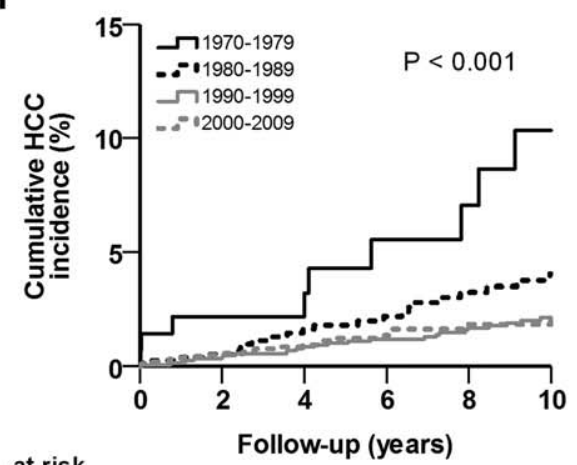

No. at risk

$\begin{array}{lllllll}1970-1979 & 139 & 112 & 93 & 74 & 61 & 49\end{array}$

$\begin{array}{lllllll}1980-1989 & 790 & 697 & 574 & 496 & 420 & 336\end{array}$

$\begin{array}{ccccccc}1990-1999 & 1520 & 1410 & 1251 & 1110 & 954 & 788\end{array}$

$\begin{array}{lllllll}2000-2009 & 1666 & 1471 & 1121 & 742 & 451 & 194\end{array}$

iii

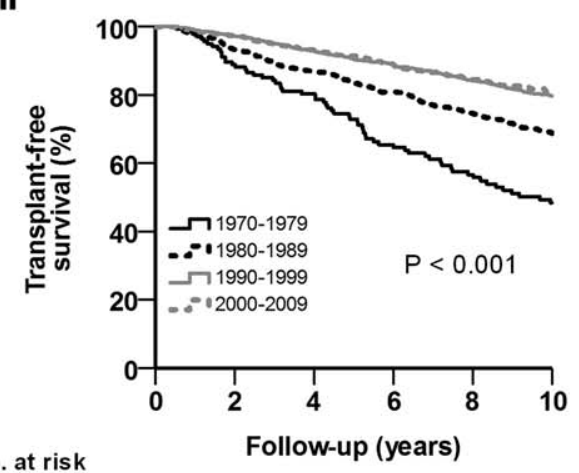

No. at risk

1970-1979

1980-1989

1990-1999

2000-2009

$\begin{array}{cccccc}143 & 117 & 99 & 78 & 63 & 51 \\ 858 & 763 & 641 & 557 & 472 & 379 \\ 1754 & 1641 & 1466 & 1315 & 1101 & 876 \\ 1815 & 1611 & 1242 & 829 & 502 & 224\end{array}$

B Adjusted

i

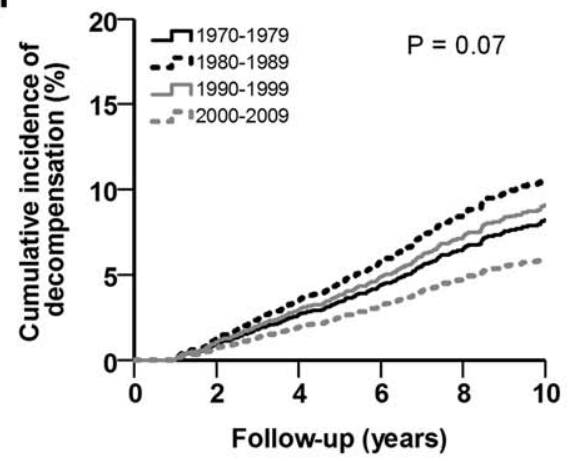

ii

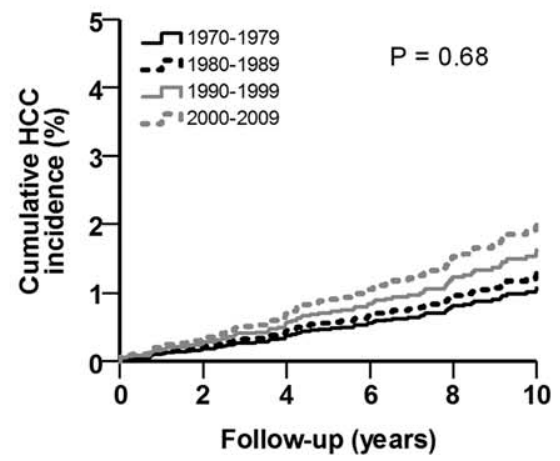

iii

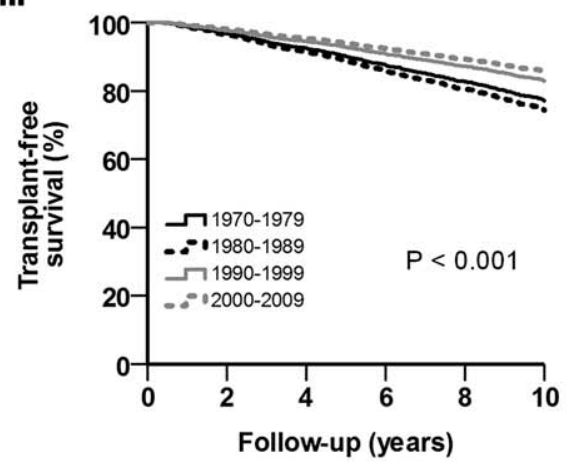

FIG. 4. Time-to-event analyses of decompensation, HCC, and liver transplantation or death over calendar time. (A) Kaplan-Meier (crude) and (B) Multivariable Cox regression (adjusted) estimates of (i) cumulative incidence of decompensation, (ii) cumulative incidence of HCC, and (iii) transplant-free survival. 
detect the disease at an earlier stage, we speculate that any changes in AMA testing have not had a major impact in the observed temporal trends. The conventional method of AMA detection is indirect immunofluorescence, yet there has been an increase in enzyme-linked immunosorbent assay-based assays and immunoblotting that have led to greater sensitivity and specificity. ${ }^{(37)}$ These improvements would translate to an increase in the proportion of AMA-positive patients; however, this has remained unchanged.

We demonstrate a 10 -year increase in the mean age at diagnosis from 1970 to 2014. A similar increase was reported in a Canadian $\mathrm{PBC}$ population, in which prevalent cases in 1996 had a median age of 53, whereas prevalent cases in 2002 had a median age of 57. ${ }^{(18)}$ These numbers coincide with the findings from our study, in which the mean ages at diagnosis in the 1990s and 2000s are 52.8 and 55.0 years, respectively. Furthermore, an increased proportion of patients diagnosed in recent years are over 50 years of age and account for $71.5 \%$ of patients diagnosed on 2010 and beyond. Comparable results were found within the UK-PBC cohort, in which $75 \%$ of patients prevalent between 2008 and 2010 were over 50 years of age. ${ }^{(38)}$

The increase in age may be attributed to the general aging of the population as the median age in North America and Europe has reportedly increased from 30 in 1970 to 40 in $2015 .^{(39)}$ This represents a 10-year increase over a 45 -year period, which is similar to the 10 -year increase in age at diagnosis we observed over a 44-year interval. Furthermore, the 34\% absolute increase of PBC patients 50 years old and above from 1970 to 2014 was greater than that of the general population, which was only $11 \%$ (25\% in 1970 to $36 \%$ in 2015). ${ }^{(39)}$ The increase in age may also be attributed to differences in the trigger for a PBC diagnosis over the years. Although we are not able to assess the symptoms in our cohort, we speculate that patients in recent decades are predominantly asymptomatic and are therefore diagnosed when they see their physician to undergo routine testing of liver function, which occurs more frequently in older individuals. Conversely, younger patients in earlier decades were more likely to develop symptoms, which led to their diagnoses. ${ }^{(40,41)}$ Lastly, the increase in age may be disease-specific and represents a shift in the natural history of PBC toward a new older at-risk population, considering that the increase in age was observed irrespective of biochemical disease stage. It can also be speculated that the later onset is a result of a prolonged subclinical disease period and potentially a delayed exposure to an unknown environmental trigger due to temporal changes in lifestyle.

An older age at diagnosis is clinically important because it has been associated with an increased likelihood of meeting Paris-I criteria for response to UDCA. ${ }^{(38)}$ Similarly, we found an older age at diagnosis to be an independent predictor of Paris-I response, yet calendar time was not a significant predictor. These results indicate the increase in age at diagnosis may be an important factor contributing to the increase in UDCA response rather than calendar time itself. Furthermore, the low response rates observed in earlier decades can be a result of inadequate UDCA dosages and the delay in treatment. The importance of an adequate UDCA dosage of $13-15 \mathrm{mg} / \mathrm{kg} /$ day has been emphasized in a study that found that $40 \%$ of UDCA nonresponders in whom the dosage was increased became responders. ${ }^{(42,43)}$

In recent decades, Patients present at an older age, yet they have a milder biochemical and histological disease stage. Improved disease severity might be explained by an earlier detection of $\mathrm{PBC}$ due to improved disease awareness leading to liver function tests and AMA assays. ${ }^{(44,45)}$ The histological disease stage at diagnosis has important prognostic implications for UDCA response and survival. Advanced histological stages are associated with an increased risk of treatment failure. ${ }^{(8)}$ In addition, the survival of UDCA-treated patients in stage I/II is similar to that of an age- and sex-matched control population, while the probability of liver transplantation or death is significantly increased in patients with advanced histological stages. ${ }^{(46)}$

Although a decrease in the number of liver transplantations for PBC has been reported over the years, ${ }^{(22)}$ an improvement in transplant-free survival has not been documented. In a Canadian population-based study of patients diagnosed between 1996 and 2002, Myers et al. did not observe a significant difference in survival according to year of diagnosis. ${ }^{(18)}$ The lack of difference in survival may be attributed to the small interval of study, which only spanned 6 years. The reported increase in median age of the general population well reflects an increase in life expectancy over time ${ }^{(39)}$; therefore, transplant-free survival of $\mathrm{PBC}$ patients was compared to that of the general population. Our study showed that transplant-free survival improved over a 44-year period, even when compared to the general population, and supports its potential role in the increased prevalence of $\mathrm{PBC}$. 
The inclusion of a large population of $\mathrm{PBC}$ patients from different geographical regions, long-term followup, and broad study period are some of the strengths of our study. However, some limitations need to be considered. First, the 1970 s and 1980 s cohorts were susceptible to a delay in documentation since study entry can be many years after the date of diagnosis in these cohorts. As such, the difference in years between these two dates was included in all multivariable analyses and we assessed a subgroup of patients with a maximum 2-year difference. The same trends emerged in the subgroup analyses, thus excluding the possibility that the delay in documentation is the reason for an advanced disease in the early cohorts. Second, due to the retrospective nature of the study, biochemical data were not available for all patients and, thus, response to UDCA could not be determined for all patients. To account for missing laboratory values, all analyses were repeated in an imputed data set and revealed similar results. Lastly, the trends observed in our study cohort could not be assessed for correlations with symptom profiles or various environmental factors previously associated with $\mathrm{PBC}$, such as smoking, age at first pregnancy, and the use of hormonal replacement therapy. ${ }^{(47)}$ Even though the trends observed may be due to a selection of patients whose diagnosis is triggered by symptoms or complications in earlier decades rather than routine liver function tests as in recent decades, we describe the presenting characteristics of a typical $\mathrm{PBC}$ patient seen by physicians and how they have changed over time. The observed temporal trends warrant further investigation in other $\mathrm{PBC}$ populations to determine whether they are universally applicable and to explore the potential influence of a changing environmental trigger.

In conclusion, we demonstrate a 10 -year increase in age at diagnosis accompanied by milder disease severity at presentation of $\mathrm{PBC}$ patients. These findings provide the most comprehensive evidence of a changing natural history of $\mathrm{PBC}$ to date.

\section{REFERENCES}

1) Kaplan MM, Gershwin ME. Primary biliary cirrhosis. N Engl J Med 2005;353:1261-1273.

2) Poupon R. Primary biliary cirrhosis: a 2010 update. J Hepatol 2010;52:745-758.

3) Lindor KD, Gershwin ME, Poupon R, Kaplan M, Bergasa NV, Heathcote EJ. Primary biliary cirrhosis. Hepatology 2009;50: 291-308.
4) Pratt DS. Primary biliary cholangitis—a new name and a new treatment. N Engl J Med 2016;375:685-687.

5) Poupon RE, Lindor KD, Cauch-Dudek K, Dickson ER, Poupon R, Heathcote EJ. Combined analysis of randomized controlled trials of ursodeoxycholic acid in primary biliary cirrhosis. Gastroenterology 1997;113:884-890.

6) Corpechot C, Carrat F, Poupon R, Poupon RE. Primary biliary cirrhosis: incidence and predictive factors of cirrhosis development in ursodiol-treated patients. Gastroenterology 2002;122: 652-658.

7) Parés A, Caballería L, Rodés J. Excellent long-term survival in patients with primary biliary cirrhosis and biochemical response to ursodeoxycholic acid. Gastroenterology 2006;130:715-720.

8) Corpechot C, Abenavoli L, Rabahi N, Chrétien Y, Andréani T, Johanet $\mathrm{C}$, et al. Biochemical response to ursodeoxycholic acid and long-term prognosis in primary biliary cirrhosis. HePATOLOGY 2008;48:871-877.

9) Kuiper EM, Hansen BE, de Vries RA, den Ouden-Muller JW, Van Ditzhuijsen TJ, Haagsma EB, et al. Improved prognosis of patients with primary biliary cirrhosis that have a biochemical response to ursodeoxycholic acid. Gastroenterology 2009;136: 1281-1287.

10) Boonstra K, Beuers U, Ponsioen CY. Epidemiology of primary sclerosing cholangitis and primary biliary cirrhosis: a systematic review. J Hepatol 2012;56:1181-1188.

11) Metcalf JV, Bhopal RS, Gray J, Howel D, James OF. Incidence and prevalence of primary biliary cirrhosis in the city of Newcastle upon Tyne, England. Int J Epidemiol 1997;26:830-836.

12) Boonstra K, Kunst AE, Stadhouders PH, Tuynman HA, Poen $\mathrm{AC}$, van Nieuwkerk KM, et al. Rising incidence and prevalence of primary biliary cirrhosis: a large population-based study. Liver Int 2014;34:e31-e38.

13) Eriksson $S$, Lindgren $S$. The prevalence and clinical spectrum of primary biliary cirrhosis in a defined population. Scand J Gastroenterol 1984;19:971-976.

14) Myszor M, James OF. The epidemiology of primary biliary cirrhosis in north-east England: an increasingly common disease? Q J Med 1990;75:377-385.

15) Remmel T, Remmel H, Uibo R, Salupere V. Primary biliary cirrhosis in Estonia. With special reference to incidence, prevalence, clinical features, and outcome. Scand J Gastroenterol 1995;30: 367-371.

16) James OF, Bhopal R, Howel D, Gray J, Burt AD, Metcalf JV. Primary biliary cirrhosis once rare, now common in the United Kingdom? Hepatology 1999;30:390-394.

17) Pla X, Vergara M, Gil M, Dalmau B, Cisteró B, Bella RM, et al. Incidence, prevalence and clinical course of primary biliary cirrhosis in a Spanish community. Eur J Gastroenterol Hepatol 2007;19:859-864.

18) Myers RP, Shaheen AA, Fong A, Burak KW, Wan A, Swain MG, et al. Epidemiology and natural history of primary biliary cirrhosis in a Canadian health region: a population-based study. Hepatology 2009;50:1884-1892.

19) McNally RJ, James PW, Ducker S, Norman PD, James OF. No rise in incidence but geographical heterogeneity in the occurrence of primary biliary cirrhosis in north east England. Am J Epidemiol 2014;179:492-498.

20) Baldursdottir TR, Bergmann OM, Jonasson JG, Ludviksson BR, Axelsson TA, Björnsson ES. The epidemiology and natural history of primary biliary cirrhosis: a nationwide population-based study. Eur J Gastroenterol Hepatol 2012;24:824-830. 
21) Gross RG, Odin JA. Recent advances in the epidemiology of primary biliary cirrhosis. Clin Liver Dis 2008;12:289-303.

22) Lee J, Belanger A, Doucette JT, Stanca C, Friedman S, Bach N. Transplantation trends in primary biliary cirrhosis. Clin Gastroenterol Hepatol 2007;5:1313-1315.

23) Hirschfield GM, Mason A, Luketic V, Lindor K, Gordon SC, Mayo M, et al. Efficacy of obeticholic acid in patients with primary biliary cirrhosis and inadequate response to ursodeoxycholic acid. Gastroenterology 2015;148:751-761.

24) Christensen E, Crowe J, Doniach D, Popper H, Ranek L, Rodés J, et al. Clinical pattern and course of disease in primary biliary cirrhosis based on an analysis of 236 patients. Gastroenterology 1980;78:236-246.

25) Locke GR 3rd, Therneau TM, Ludwig J, Dickson ER, Lindor KD. Time course of histological progression in primary biliary cirrhosis. Hepatology 1996;23:52-56.

26) Lammers WJ, Hirschfield GM, Corpechot C, Nevens F, Lindor $\mathrm{KD}$, Janssen HL, et al. Development and validation of a scoring system to predict outcomes of patients with primary biliary cirrhosis receiving ursodeoxycholic acid therapy. Gastroenterology 2015;149:1804-1812.

27) Lammers WJ, Van Buuren HR, Hirschfield GM, Janssen HL, Invernizzi $\mathrm{P}$, Mason $\mathrm{AL}$, et al. Levels of alkaline phosphatase and bilirubin are surrogate end points of outcomes of patients with primary biliary cirrhosis: an international follow-up study. Gastroenterology 2014;147:1338-1349.

28) Nguyen DL, Juran BD, Lazaridis KN. Primary biliary cirrhosis. Best Pract Res Clin Gastroenterol 2010;24:647-654.

29) European Association for the Study of the Liver. EASL clinical practice guidelines: the diagnosis and management of patients with primary biliary cholangitis. J Hepatol 2017;67:145-172.

30) Ludwig J, Dickson ER, McDonald GA. Staging of chronic nonsuppurative destructive cholangitis (syndrome of primary biliary cirrhosis). Virchows Arch A Pathol Anat Histol 1978;379: 103-112.

31) Scheuer P. Primary biliary cirrhosis. Proc R Soc Med 1967;60: 1257-1260.

32) Ter Borg PC, Schalm SW, Hansen BE, Van Buuren HR. Prognosis of ursodeoxycholic acid-treated patients with primary biliary cirrhosis. Results of a 10-yr cohort study involving 297 patients. Am J Gastroenterol 2006;101:2044-2050.

33) Trivedi PJ, Bruns T, Cheung A, Li KK, Kittler C, Kumagi T, et al. Optimising risk stratification in primary biliary cirrhosis: AST/platelet ratio index predicts outcome independent of ursodeoxycholic acid response. J Hepatol 2014;60:1249-1258.

34) Kumagi $T$, Guindi $M$, Fischer SE, Arenovich $T$, Abdalian R, Coltescu C, et al. Baseline ductopenia and treatment response predict long-term histological progression in primary biliary cirrhosis. Am J Gastroenterol 2010;105:2186-2194.

35) Corpechot C, Chazouillères O, Poupon R. Early primary biliary cirrhosis: biochemical response to treatment and prediction of long-term outcome. J Hepatol 2011;55:1361-1367.
36) Bergquist A, Said K, Broomé U. Changes over a 20-year period in the clinical presentation of primary sclerosing cholangitis in Sweden. Scand J Gastroenterol 2007;42:88-93.

37) Oertelt S, Rieger R, Selmi C, Invernizzi $P$, Ansari AA, Coppel RL, et al. A sensitive bead assay for antimitochondrial antibodies: chipping away at AMA-negative primary biliary cirrhosis. Hepatology 2007;45:659-665.

38) Carbone M, Mells GF, Pells G, Dawwas MF, Newton JL, Heneghan MA, et al. Sex and age are determinants of the clinical phenotype of primary biliary cirrhosis and response to ursodeoxycholic acid. Gastroenterology 2013;144:560-569.

39) United Nations. World population prospects: the 2017 revision. https://esa.un.org/unpd/wpp/Publications/Files/WPP2017_KeyFindings.pdf.

40) Mahl TC, Shockcor W, Boyer JL. Primary biliary cirrhosis: survival of a large cohort of symptomatic and asymptomatic patients followed for 24 years. J Hepatol 1994;20:707-713.

41) Prince M, Chetwynd A, Newman W, Metcalf JV, James OF. Survival and symptom progression in a geographically based cohort of patients with primary biliary cirrhosis: follow-up for up to 28 years. Gastroenterology 2002;123:1044-1051.

42) Lammers WJ, Leeman M, Ponsioen CI, Boonstra K, van Erpecum KJ, Wolfhagen FH, et al. How the concept of biochemical response influenced the management of primary biliary cholangitis over time. Neth J Med 2016;74:240-246.

43) Angulo P, Dickson ER, Therneau TM, Jorgensen RA, Smith C, DeSotel CK, et al. Comparison of three doses of ursodeoxycholic acid in the treatment of primary biliary cirrhosis: a randomized trial. J Hepatol 1999;30:830-835.

44) Prince MI, Chetwynd A, Craig WL, Metcalf JV, James OF. Asymptomatic primary biliary cirrhosis: clinical features, prognosis, and symptom progression in a large population based cohort. Gut 2004;53:865-870

45) European Association for the Study of the Liver. EASL clinical practice guidelines: management of cholestatic liver diseases. J Hepatol 2009;51:237-267.

46) Corpechot C, Carrat F, Bahr A, Chrétien Y, Poupon RE, Poupon R. The effect of ursodeoxycholic acid therapy on the natural course of primary biliary cirrhosis. Gastroenterology 2005; 128:297-303.

47) Gershwin ME, Selmi C, Worman HJ, Gold EB, Watnik M, Utts J, et al. Risk factors and comorbidities in primary biliary cirrhosis: a controlled interview-based study of 1032 patients. Hepatology 2005;42:1194-1202.

Author names in bold designate shared co-first authorship.

\section{Supporting Information}

Additional Supporting Information may be found at onlinelibrary.wiley.com/doi/10.1002/hep.29717/suppinfo. 